\section{Multifunctional Conjugated Polymer Nanoparticles (CPNs) for Biological Applications}

\author{
Mark Green, ${ }^{1,2}$ Dermott O'Callaghan ${ }^{2}$
}

I) Department of Physics, King's College London, The Strand, London, WC2R 2LS, UK. 2) Stream Bio Ltd., NETpark Incubator, Thomas Wright Way Sedgefield, Stockton-On-Tees TS2I 3FG, UK.

\section{Abstract}

Luminescent markers for monitoring biological processes is a well-developed, although imperfect science. Luminescence, in this case, is actually photoluminescence from an organic semiconducting electronic structure, where a photo-excited electron recombines with a photo-generated hole, specifically from an exciton in the pi/pi* orbitals.

Organic dyes are the usual material when cell-imaging studies (or associated diagnostic procedures) are required. Recent advances in material science have opened up new avenues to biologically-relevant luminescent materials, usually considered the domain of organic chemists. These new materials exploit the robust optica properties required for display applications and when combined with other functionalities provided by such materials, represent the next generation of imaging technologies

The emergence of chemically-accessible nanotechnologies over the last 20 years has opened up a new family of biologically-active materials. The interaction of gold nanoparticles with DNA might be considered one of the first examples of the crossover between solid-state sciences with biology, two disciplines that traditionally have not overlapped. The comparative sizes of, for example, proteins and nanoparticles however make the merging of such technologies obvious. These initial developments inspired further work into new multidisciplinary arenas and one of the most successful might be considered the development of new imaging agents for biology, with semiconductor quantum dots (QDs) notably emerging from typical solid-state applications (such as display devices and solar cells) to become commercially available cellular imaging agents. The cross-over from physics and materials chemistry to biology allowed medica scientists to access the positive optical attributes of inorganic semiconducting nanomaterials - notably the wide range of available wavelengths, from UY, through the visible spectrum to near IR.This tuneable bright emission, explained by quantum mechanical concepts such as the confinement of charge carriers in a potential-well, coupled with narrow optica emission profiles allowed new imaging agents with relatively long-term stability. Their broad absorption profiles also allowed multiplexing of, for example, multiple markers [I].This paradigm shift in medical imaging is not without limitations. QDs are typically based on cadmium and even the next-generation cadmium-free QD materials have limitation base on their constituent elements and this potentially hampers their eventual clinical use.There have been many studies on the potential toxicity of quantum dots, and whilst reports have highlighted that the amount of cadmium used in such studies are well technologies lock such elements inside the particle, questions regarding the use and disposal of such materials remain. [2]

Other nanomaterials, such as carbon nanotubes also exhibit positive features not normally associated with typical molecular fluorescent imaging agents, notably in near-IR imaging technologies where below typical exposure limits and that efficient shel

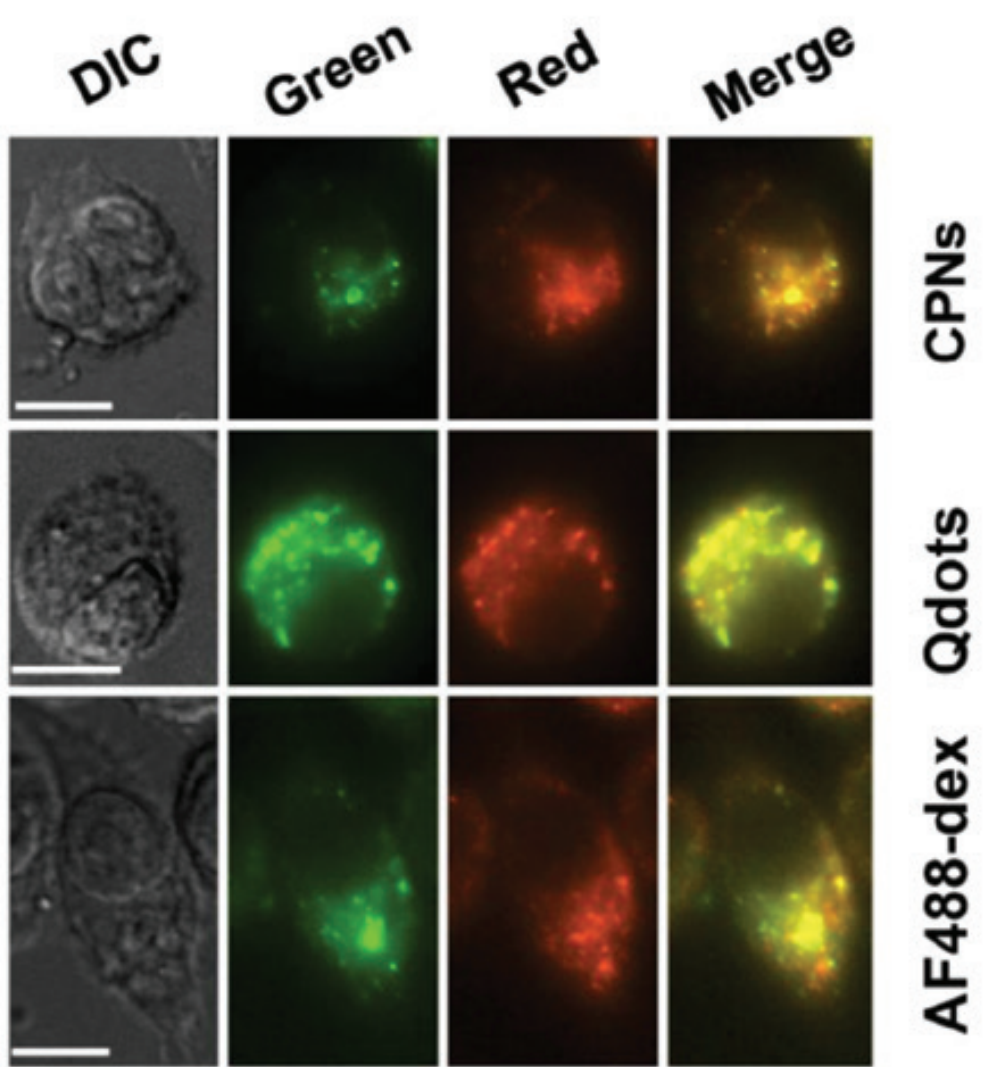

Figure $I-$ Comparison of $J 774$ A. I cells after incubations with conjug
Scale bar $=10 \mu m(5)$. Figure used with permission from Springer.

the use of purely organic materials is highly visible spectrum is now commercially available. attractive. For example, Hong et al. reported However, the majority of these materials, having impressive imaging capabilities in the brain using been designed for device applications, are soluble carbon nanotubes injected into the tail-vein of a only in organic solvents and are therefore limited mouse [3]. Other carbon-based materials, when as biological reagents. Water-soluble conjugated processed into nanoscale materials, exhibit optical polymers are now emerging in the literature and and physical properties that potentially surpass have become commercially available, yet the full other imaging technologies. Conjugated polymers, palette of colours available for display applications initially developed as display materials, have notable have not translated through to their aqueous properties that make them appealing for imaging analogues. One simple way to overcome such [4]. Their inherent stability, essential for display limitations is to take the available polymers and applications, make them ideal for imaging, as the process them into passivated conjugated polymer majority of luminescent small molecules have nanoparticles (CPNs). Using this technology, one can limited photostability. The chemistry behind their take readily-available materials covering a number of optical properties is now well-developed, and a wavelengths and process them into a water-soluble wide range of materials with emission across the form that retains a high degree of their original 


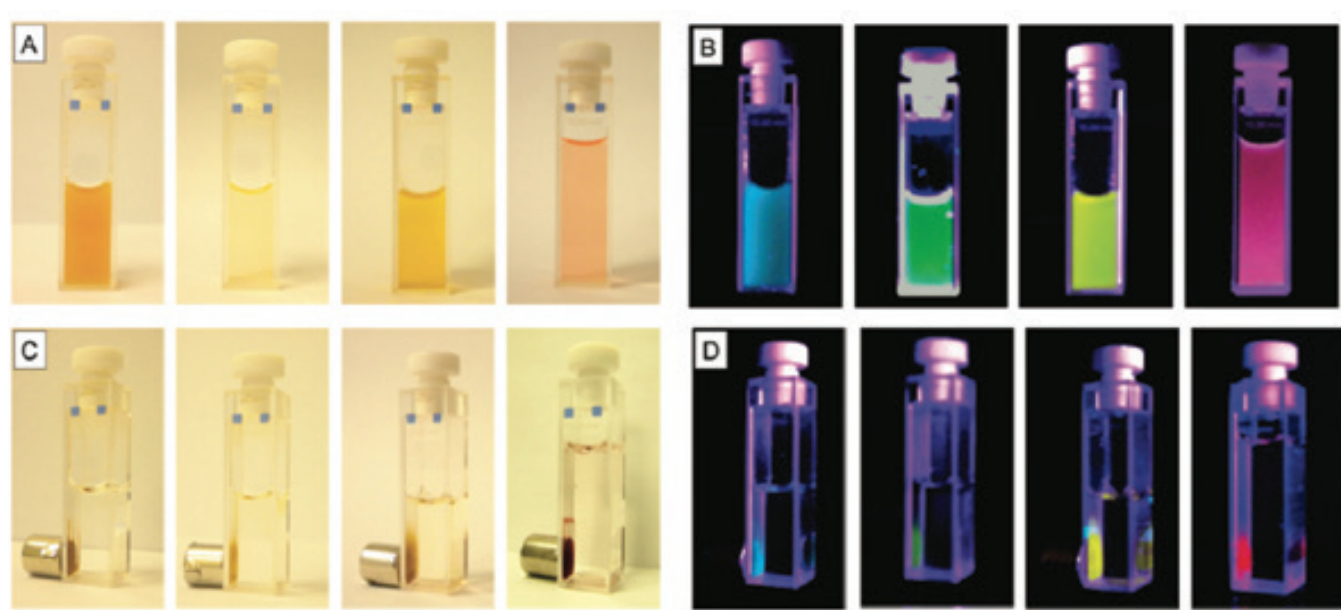

Figure 2 -A selection of magnetic conjugated polymer nanoparticles, illuminated at $365 \mathrm{~nm}$ next to a magnet. Reprinted (adapted) with permission from P. Howes, M. Green, A. Bowers, D. Parker, G. Varma, M. Kallumadil, M. Hughes, A. Warley, A. Brain, R. Botnar, JAm. Chem. Soc,., 2010,

emissive properties. Whilst a reduction in emission quantum yield occurs, the resulting nanoparticles remain remarkably bright and photostable, with most conjugated polymer nanoparticles preserving their optical properties for months, if not years in ambient conditions. Whilst the emissive properties of CPNs are impressive in a standard laboratory setting, it does not necessarily translate to biologica samples. A study of simple conjugated polyme nanoparticles composed of the emitting polyme PFBT with a polyethylene glycol surface passivation compared to QDs and dye molecules in J774A. cells highlighted that the polymer particles were significantly brighter whilst in the cell, allowing for less material to be used (figure I) [5]. It was also highlighted that the polymer particles displayed no evidence of toxicity, a major perceived drawback of the use of QDs. The reported lack of toxicity is a major benefit of CPN use. Whilst the concept of nanotoxicology remains an emerging issue and set protocols are yet to be described, the use of organic materials over heavy metal inorganics is a distinct benefit, if not only for waste disposal in a clinical setting. Another obvious advantage of encapsulating conjugated polymers in a biologically compatible shell (usually an amphiphilic polymeric species although phospholipids or proteins have also been used) is the fact that a wide range of species are available. This provides numerous linking chemistries to facilitate attachment of antibodies, proteins, etc without the direct attachment to the emitting conjugated polymer, which could have a detrimental effect on the optical properties. This separation of capping/linking moiety and emitting core provides numerous combinations of emitting colours and targeting functionalities, including simple PEGylated surfaces, for biokinetic studies.

Another obvious difference between other nanomaterials systems and CPNs is their actual structure; CPNs are prepared by collapsing a hydrophobic polymer chain into a nanoparticle in the presence of a capping agent. Whilst this process forms the nanoparticle, no bonds are actually formed or broken and the formation of the nanoparticle is almost entirely a steric event. The interaction of chains in close proximity to one another can in some cases induce shifts in the optical properties without changing the actual chemical structure. Whilst this simplifies the formation of the materials, it also provides an additional feature not usually available in other materials (at least not in the same manner); that of incorporating other materials into the nanoparticle during the polymer collapsing event, resulting in multifunctional materials.

The obvious and most effective multifunctional CPN is the magnetic/luminescent material, maintaining the emissive properties of the conjugated polymer, the biologically active surface combined with the MRI-active and magnetic functionality provided by the addition of superparamagnetic $\mathrm{Fe}_{3} \mathrm{O}_{4}$ into the hydrophobic emitting polymer phase [6] as shown in figure 2. Surprisingly, whilst the addition of iron oxide nanoparticles with a broad absorption profile reduced the emission quantum yield of the final material, the emission was still remarkably bright and no noticeable difference was observed with their use in cell imaging. The main advantage of the addition of the magnetic particles was the additional magnetic resonance imaging (MRI) functionality, making a dual multimodal imaging probe. The formation of the nanoparticles might be considered to be more processing than chemistry, having more in common with electronic device manufacture than traditional semiconductor doping. Numerous other materials can be processed into the body of the particle, further enhancing the optical properties or providing other functionalities.

Magnetic 'dopants', that provide the MRI active element do however have a limitation: the iron oxide reduces the brightness of the materials and provides only negative contrast in MR imaging. The use of a gadolinium chelate as the surfactant instead of iron oxide resulted in maintaining the inherent bright emission whilst providing positive contrast in MR imaging [7] with relaxation times calculated (figure 3). Multimodal imaging is an emerging technique in medicine, allowing a single scan to take advantage of the numerous imaging technologies resulting in faster and more accurate diagnosis and the associated benefit to patients. The preeminent multimodal imaging technology is the combination of $\mathrm{MRI}$ and positron emission tomography (PET), with clinical PET/MR scanners now commercially available, notably the Philips Ingenuity TF whole body PET/MR Imaging system, which has overcome substantial engineering issues associated with merging the two differing technologies in a single platform. It is worth noting that to date, there re no clinical optical/MR scanners available. One further advantage of the incorporation of magnetic particles is the potential for magnetic purification, using a bar magnetic to isolate the particles, removing the nanomaterials from unbound antibodies, for example.

\section{Bio-targeting of CPNs}

In order to utilise CPNs for applications in biology it is necessary to engineer the CPNs to selectively identify molecular targets of interest, such as biomarkers and cell type identifiers. The targeting mechanism can range from the physiochemical ature of the CPN itself to the specific attachment of specialised biomolecules, such as antibodies or their synthetic equivalents, to the CPN. There are also a number of options for the attachment of these targeting components, as they can be bound directly to the conjugated polymer or to the coating surfactant of the CPN and they can be attached prior, during or after the formation of the CPN.The optimal design principles for bio-targeting of CPN are still emerging with innovative and alternative approaches being developed across a large number of groups and a number of approaches that have been successful are described below.

CPNs have been designed that are of a size (IO$100 \mathrm{~nm}$, with no magnetic or multifunctional additional structure) and surface charge (negative) that are readily taken up by mammalian cells via endocytosis [8]. The uptake of CPN by endocytosis was studied in J774A.I cells, with the CPN shown to move through the endocytotic pathway to the ysosomes, tracking with Texas Red labelled dextran and ultimately co-localising with LAMP-I in a perinuclear distribution [9]. The intense brightness of the CPN allowed loading concentrations as low as $155 \mathrm{pM}$ (270 ppb) to be detected [9].

Proteins, and in particular antibodies, are widely used for targeting as they have high specificity and affinity for molecules of interest. A range of approaches have been used to couple proteins to 
CPNs with a particular focus on optimising the protein to CPN stoichiometry and ensuring that the coupled protein remains active and sterically free to bind its target [10]. The targeting protein can be covalently bonded to the polymer side chains or to the detergent surface coating of the CPN. Numerous groups have successfully used side chain covalent bonding, either directly or via a streptavidin/biotin coupling to attach antibodies an label specific target proteins in a range of cell types. For example, I-ethyl-3-(3-dimethylaminopropyl) carbodiimide hydrochloride (EDC) has been use to couple anti-tubulin and anti-CD326 EpCAM antibodies, via streptavidin/biotin, to carboxy groups on the polymer chains of their CPN [1 I, I2] These antibody-labelled CPNs successfully labelled HeLa and MCF-7 cells, highlighting both membrane surface and intracellular target proteins. Feng et al [13] used EDC coupling to attach anti-EpCAM and anti-ErbB2 antibodies to the poly(styrene-co-malei anhydride) (PSMA) surface of CPNs and utilised them to differentiate between SK-BR3, MCF-7 an HeLa cells. StreamBio have also successfully attached streptavidin to the surface of their CPN, utilisin them in fluorescent plate-based assays and to labe biotinylated antibodies for molecular and cellular detection.

CPN have properties that make them extremely durable (photostable, thermostable, $\mathrm{pH}$ resistant), which are not matched by naturally occurring protein targeting molecules. This has led to interest an research in coupling CPNs with synthetic targetin molecules that are more robust and do not require specialist handling/storage. Synthetic targeting molecules are derived from a number of sources (aptamers - nucleotide sequences, affimer an affibodies - stabilised protein structures, molecula imprinted polymers - organic polymers) and can be readily attached to CPNs. Click chemistry has been used to attach an anti-HER2 affibody to the CPN PEG matrix, which were able to identify HER2 overexpressing SKBR-3 breast cancer and HepG2 live cancer cell lines $[14,15]$.These stable CPN-synthetic targeting molecule particles were sufficiently robust and bright to be able to monitor liver tumour growth in vivo for up to 27 days.

CPNs can also be targeted using small molecules attached to their surface.The targeting of CPN using the drug Plerixafor has been reported, which bind CXCR4 [16].The CPN-Plerixafor particles bound to CXCR4 and prevented CXCR4 from internalising showing that the drug retained its inhibitory function. Folate has been used by a number of groups to target CPN to cancerous cell types overexpressing the folate receptor (e.g. ovarian, breast, brain and lung cancer) $[12,17,18]$

\section{Fluorescent microscopy}

The bright fluorescence, robustness an multimodality of the CPN make them highly utilisable across numerous applications in bioscience and in particular microscopy. CPNs offer exciting advantages in the field of fluorescent microscopy where their high-performance characteristics enable both higher resolution of signal and greater sensitivity of detection. As discussed above, CPN can be readily attached to a wide range of targeting molecules, allowing them to label proteins of interest both intracellularly and on the cell surface. This has been demonstrated across a wide range of proteins, cell types and tissues $(11,13,17)$. The larger size of the CPN compared to traditional fluorophores had led to some concern around access to some intracellular structures even once cells are permeablised, however CPNs with ultrasmall diameters $(3.0-4.5 \mathrm{~nm})$ have been reported which can overcome this issue [10]. The brightness of the CPN allows studies to be conducted using low levels of illumination reducing damage to samples and background interference. This allows for imaging of samples over days with stable fluorescence. The brightness of the CPNs also enables high speed kinetic studies requiring rapid image capture rates and short exposure times. The intensity of the CPN signal allows individual particles to be observed using standard fluorescent light microscopy ensuring

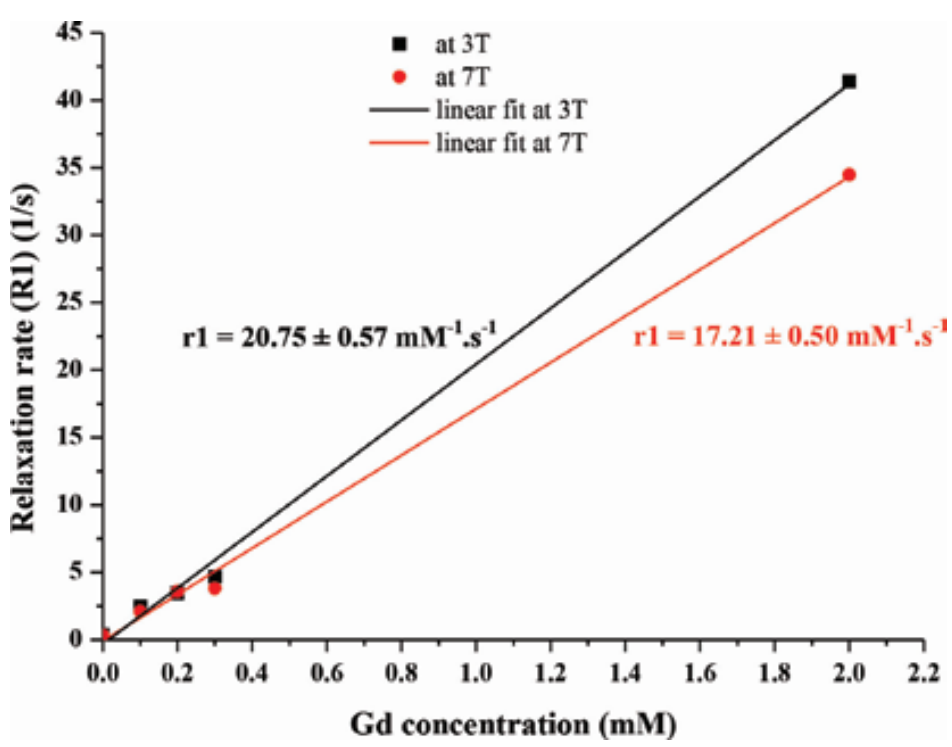

Figure 3 - Relaxation times $\left(R_{1}\right)$ versus $G d$ concentration for a conjugated polymer/Gd lipid conjugate. Used with permission.

that even very low levels of target molecule can be detected [10].

The robustness of the CPN means that labels will not photobleach during prolonged or high intensity illumination and do not fade during storage. The CPN fluorescence is also unaffected by fixation in formaldehyde and other standard fixation techniques allowing CPN labelling to take place in vivo and biopsy and histological samples to be processed using standard methods while retaining a fluorescent signal [7]. The stability of the CPN also allows them to be used as reference markers and calibration beads allowing comparisons between mages over time and under differing conditions.

There is a wide range of conjugated polymers available that can be used in the production of $\mathrm{CPN}$, allowing for great diversity in the spectral properties. Authors have reported numerous CPNs with excitation and emission peaks across the visible spectrum [19]. CPNs produced to date are frequently selected to have spectra that match those of existing fluorophores and their associated filter sets to facilitate their validation in standard applications and comparison to existing labels. The diversity of available polymers also allows CPNs with novel spectral properties to be readily produced to utilise gaps in the current palette of fluorophores and excitation wavelengths. StreamBio are currently developing a CPN with an $808 \mathrm{~nm}$ excitation and near-IR emission. Additionally, CPN have been identified that are compatible with 2 photon excitation and emission in the infrared tissue window, allowing optical slices to be taken at depths up to a millimetre $[20,21]$. CPNs, while relatively large, have an intensity and stability that offers opportunities in the field of super resolution microscopy [22].

\section{Flow cytometry}

The brightness of the CPN has the potential to make a significant impact on flow cytometry and flourescence-activated cell sorting (FACS) allowing surface receptors and biomarkers expressed at very low levels to be labelled and reliably sorted [13,23]. Minimal level of detection of a simple, non-magnetic CPN in a flow cytometer has been determined to 19pM (86 ppb) compared to 980pM for a QDot or 11.2nM for AF488-Dex [9]. The stability of the CPN facilitates sample collection, as they do not require specialised storage and are compatible with sample preparation processes $(\mathrm{pH}$. Temperature etc.). Magnetically enabled CPN, such as those from StreamBio, can enrich rare cell types in samples by binding to specific surface epitopes and allowing 
labelled cells to be separated using a magnet. The ability to select specific conjugated polymers for the excitation and emission spectra and readily transform them into CPN allows novel fluorochromes to b generated to fit into existing spectral gaps and to utilise emerging excitation sources and laser lines. This can significantly increase the number of analysis channels available for panel studies.

\section{Tissue imaging}

CPNs that have fluorescent excitation and emission peaks in the near or far tissue window have great potential in surgical application where labelling of specific tissue types is required, such as tumour margin imaging. The intense brightness of the CPN ensures that diseased and healthy tissue can be readily differentiated, and the maximum amount of tumour removed while sparing other tissues. Excitation/emission in the $650-1350 \mathrm{~nm}$ range allows labelled tissue to be visualised using standard surgical theatre apparatus. CPNs carrying additiona particles, such as iron oxide or gadolinium, can b visualised using MRI prior to surgery to facilitate preparation and planning for the procedure. The biocompatibility and non-toxic nature of the CPN are further advantages for tissue labelling $[14,15$, $17,24]$

\section{Molecular biology, \\ pharmaceutical assays and diagnostics}

Fluorescent labels are widely used for numerous molecular biology applications. Western blotting DNA detection arrays, fluorescent ELISA, qPCR etc. and CPNs are highly compatible and readily substitutable into these applications bringing the superior performance characteristics of the CPN [25]. The brightness of the CPN increases the sensitivity of the applications and the signal has a linear relationship with the quantity of analyte unlike that seen with enzymatic detection methods. As with flow cytometry, the CPNs are compatible with challenging sample collection and preparation conditions, increasing the range of available sampling approaches. These characteristics are of particula interest for rapid diagnostic tests (RDT) applicable in challenging environments where refrigeration and specialist reader technologies are not accessible [26]. These RDT would be of particular use to diagnose and guide treatment for diseases such as malaria, HIV and hepatitis C. The multiple emission colours available would allow for tests that can diagnose multiple diseases or disease strains from a single sample $[27,28]$.

Simple CPNs are not restricted to imaging modalities the use of a conjugated polymer particle as a carrie for drugs introduces the option of theranostic nanoparticles, allowing imaging of tumours and the simultaneous delivery of a therapeutic payload, for example. The advantages of a nanoparticles system to deliver drugs to a tumour is twofold; the particle can be used to deliver hydrophobic drugs that would not normally be available, and nanoparticles can also exploit the EPR (enhanced permeability and retention) effect where particles can access tumours through the leaky vasculature. It should be noted that this is far from accepted; the EPR effect remains controversial and a detailed recen report highlighted that only $0.7 \%$ of administered nanoparticles access tumours [29].

There are still unresolved issues that need to be addressed - the toxicology of nanomaterials and associated standards are still undefined, although all research work to date appears to indicate conjugated polymers are inert and present no issues. Structurally, nanomaterials should be perfectly monodispersed and be passivated with a known number of surface molecules - ideally a single linking moiety, with a standard brightness that does not fade. There is no consensus on the ideal particle size; certain materials will need to be renally cleared, whilst other applications impose no such limits. In real life, no nanomaterial successfully addresses all these requirements, and one must pick and choose the material for the application. However, conjugated polymer nanoparticles provide bright emission over a wide spectral wavelength with an enhanced non-toxic stability that can withstand almost indefinite storage in ambient conditions, which can further be coupled with a magnetic handle that allows separation and purification.

In this brief review, we have highlighted an emerging imaging system inspired by solid-state materials science that also provides a wealth of further potential biological advances, which could ultimately find acceptance in clinical settings.

\section{References}

[1] Lee-Montiel, F. T., Li, Peter., Imoukhuede 2015. "Quantum dot multiplexing for the profiling of cellular receptors." Nanoscale 7: 18504-18514.

2] Bottrill, M., Green, M. 201I. "Some aspects of quantum dot toxicity'. J. Chem. Soc., Chem. Comm. 47, $7039-7050$.

[3] Hong, G., Diao, S, Chang, J., Antaris, A. L., Chen, C., Zhang, B., Zhao, S., Atochin, D. N., Huang, P. L., Andreasson, K. I., Kuo, C. J., Dai, H. 2014. “Throughskull fluorescence imaging of the brain in a new nearinfrared window." Nature Photonics 8: 723-730.

[4] Tuncel, D., Volkan Demir, H. 2010. 'Conjugated polymer nanoparticles". Nanoscale 2: 484-494.

[5] Fernando, L. P., Kandel, P. K., Ackroyd, P. C., Christensen, K. A. 2012. "The relative brightness of PEG lipid-conjugated polymer nanoparticles as fluidphase markers in live cells." Anal. Bioanal. Chem. 404 (I0): 3003-30I4.

[6] Howes, P., Green, M., Parker, D.,Varma, G., Kallumadil, M., Hughes, M., Warley, A., Brain, A., Botnar, R. 2010. "Magnetic conjugated polymer nanoparticles as bimodal imaging agents." J.Am. Chem. Soc. 132: 98339842

[7] Hasim, Z., Green, M., Chung, P. H., Suhling, K., Protti, A, Phinikaridou, A, Botnar, R, Ahmad Khanbeigi, R. y. A Commander $N$, Rowland, $C$ Scott, J., Jenner, D. 2014, “Gd-Containing Conjugated Polymer Nanoparticles: Bimodal nanoparticles for Fluorescence and MRI Imaging." Nanoscale 6: 8376-

[8] Oh N and Park J.2014."Endocytosis and exocytosis of nanoparticles in mammalian cells" Int J Nanomedicine. 2014; 9 (Suppl I): 5I-63.
2] Lawrence P. Fernando, Prakash K. Kandel, Jiangbo Yu, Jason McNeill, P. Christine Ackroyd, and Kenneth A. Christensen. 2010. "Mechanism of Cellular Uptake of Highly Fluorescent Conjugated Polymer Nanoparticles" Biomacromolecules, II (10): 2675 2682

[10] Lia, K., Liu, B. 20I4. "Polymer-encapsulated organic nanoparticles for fluorescence and photoacoustic imaging" Chem. Soc. Rev., 43: 6570.

[II] Chen, C.-P.; Huang, Y.-C.; Liou, S.-Y.; Wu, P.-j; Kuo, S.-Y.; Chan, Y.-H. 2014. "Near-Infrared Fluorescent Semiconducting Polymer Dots with High Brightness and Pronounced Effect of Positioning Alkyl Chains on the Comonomers." ACS Appl. Mater. Interfaces 6: 21585-21595.

[12] Liu, H.-Y.;Wu, P.-..; Kuo, S.-Y.; Chen, C.-P.; Chang, E.H.; Wu, C.-Y.; Chan, Y.-H. 2015. "Quinoxaline-Based Polymer Dots with Ultrabright Red to Near-Infrared Fluorescence for In Vivo Biological Imaging." J. Am. Chem. Soc. 137: 10420-10429.

[13] Feng, L.; Liu, L.; Lv, F.; Bazan, G.C.; Wang, S. 2014. "Preparation and Biofunctionalization of Multicolor Conjugated Polymer Nanoparticles for Imaging and Detection of Tumor Cells": Adv. Mater. 26: 39263930

[4] Liu, J.: Feng, G: Ding, D.; Liu, B. 2013، "Bright FarRed/Near-Infrared Fluorescent Conjugated Polymer Nanoparticles for Targeted Imaging of HER2-Positive Cancer Cells." Polym. Chem. 4: 4326-4334.

[15] Liu, J.; Li, K.; Liu, B. 2015. "Far-Red/Near-Infrared Conjugated Polymer Nanoparticles for Long-Term In Situ Monitoring of Liver Tumor Growth." Adv. Sci. 5: 1500008.

[16] Li, M.; Nie, C.; Feng, L.; Yuan, H.; Liu, L.; Lv, F.; Wang, S. 2014. "Conjugated Polymer Nanoparticles for Cell Membrane Imaging." Chem. Asian 1. 9: 312I-3124.

[17] Ding, D.; Liu, J.; Feng, G.; Li, K.; Hu, Y.; Liu, B. 2013. "Bright Far-Red/Near-Infrared Conjugated Polymer Nanoparticles for In Vivo Bioimaging." Small 9: 30933102

18] Ahmed, E.; Morton, S.W.; Hammond, P.T.; Swager, T.M. 2013. "Fluorescent Multiblock -Conjugated Polymer Nanoparticles for In Vivo Tumor Targeting." Adv. Mater. 25: 4504-45I0.

[19] Braeken Y, Cheruku S, Ethirajan A, Maes W. 2017. "Conjugated Polymer Nanoparticles for Bioimaging." Materials (Basel). 10(12):1420.

[20] Wu, C.; Szymanski, C.; Cain, Z.; McNeill, J. 2007. 
"Conjugated Polymer Dots for Multiphoton Fluorescence Imaging." J. Am. Chem. Soc. I29: I2904 12905.

[2I] Pecher, J.; Huber, J.; Winterhalder, M.; Zumbusch, A. Mecking, S. 2010. "Tailor-Made Conjugated Polymer Nanoparticles for Multicolor and Multiphoton Cell Imaging." Biomacromolecules II: 2776-2780.

[22] Jiang, Y., Novoa, M., Nongnual, T., Powell, R., Bruce, T. McNeill, J. 2017. "Improved Superresolution Imaging Using Telegraph Noise in Organic Semiconductor Nanoparticles", Nano Letters 17(6):3896-390 I.

[23] Sun, M., Sun, B., Liu, Y., Shen, Q.-D. Jiang, S. 2016. "Dual-Color Fluorescence Imaging of Magnetic Nanoparticles in Live Cancer Cells Using Conjugate Polymer Probes". Scientific Reports. 6. 22368.

[24] Bruns, Oliver T., Thomas S. Bischof, Daniel K. Harris, Daniel Franke, Yanxiang Shi, Lars Riedemann, Alexander Bartelt, 2017. "Next-Generation in Vivo Optical Imaging with Short-Wave Infrared Quantum Dots." Nature Biomedical Engineering I (4): 0056.
[25] Debbage P. Jaschke W. 2008. "Molecular imagin with nanoparticles: giant roles for dwarf actors." Histochem Cell Biol. I30(5):845-75.

[26] Lyberopoulou, A., Efstathios P. Efstathopoulos and Maria Gazouli (September 7th 2016). Nanotechnology-Based Rapid Diagnostic Tests, Proof and Concepts in Rapid Diagnostic Tests and Technologies, Shailendra K. Saxena, IntechOpen, DOI 10.5772/63908.

[27] Quesada-González D, Merkoçi $\quad$ A. 2018 "Nanomaterial-based devices for point-of-care diagnostic applications." Chem Soc Rev. 47(13):46974709.

[28] Quesada-González D, MerkoçiA. 2015."Nanoparticlebased lateral flow biosensors." Biosens Bioelectron. 73:47-63.

[29] Wilhelm, S., Tavares, A. J., Dai, Q., Ohta, S., Audet, J., Dvorak, H. F., Chan, W. C. W. 2016, "Analysis of nanoparticle delivery to tumours." Nature Rev. Materials I: 16014

\section{Mark Green}

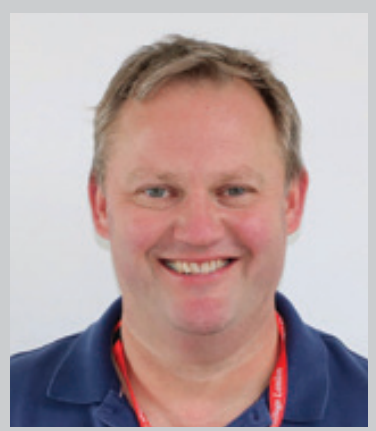

Prof.Mark Green (MG) is a Professor of Nanotechnology with experience in the synthesis of nanomaterials. He has previously worked in industry, developing nanomaterials for imaging applications and initiated the synthetic routes to multimodal conjugated polymer systems that were adopted by Stream Biosciences, which won an Institute of Physics startup-of the year 2018. He has been awarded several EPSRC responsive mode and platform grants covering the preparation of nanomaterials for display applications and biological imaging.

\section{Dermott O'Callaghan}

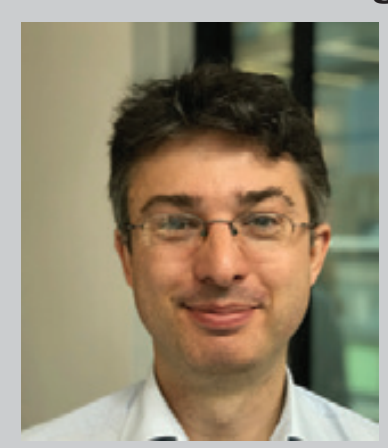

Dermott is a molecular biologist with over 16 years experience in the pharmaceutical industry working for AstraZeneca, Pfizer and as an independent consultant. During this time he gained extensive scientific and technical expertise in all stages of product development, project management and drug discovery, from target identification to candidate drug selection.

His focus has been on the application of novel technologies to drug discovery, with an emphasis on microscopy, fluorescent and electrophysiological approaches. Dermott's in-depth knowledge of numerous drug discovery techniques, in-vitro processes, and applications of the latest industry technology within the field of molecular biology is now being applied to product definition of Stream's core technology.

\section{FluidFM ${ }^{\circledast}$ probe microscopy}

Study individual cell properties

Manipulate cells without destroying them

Inject into or extract from live cells
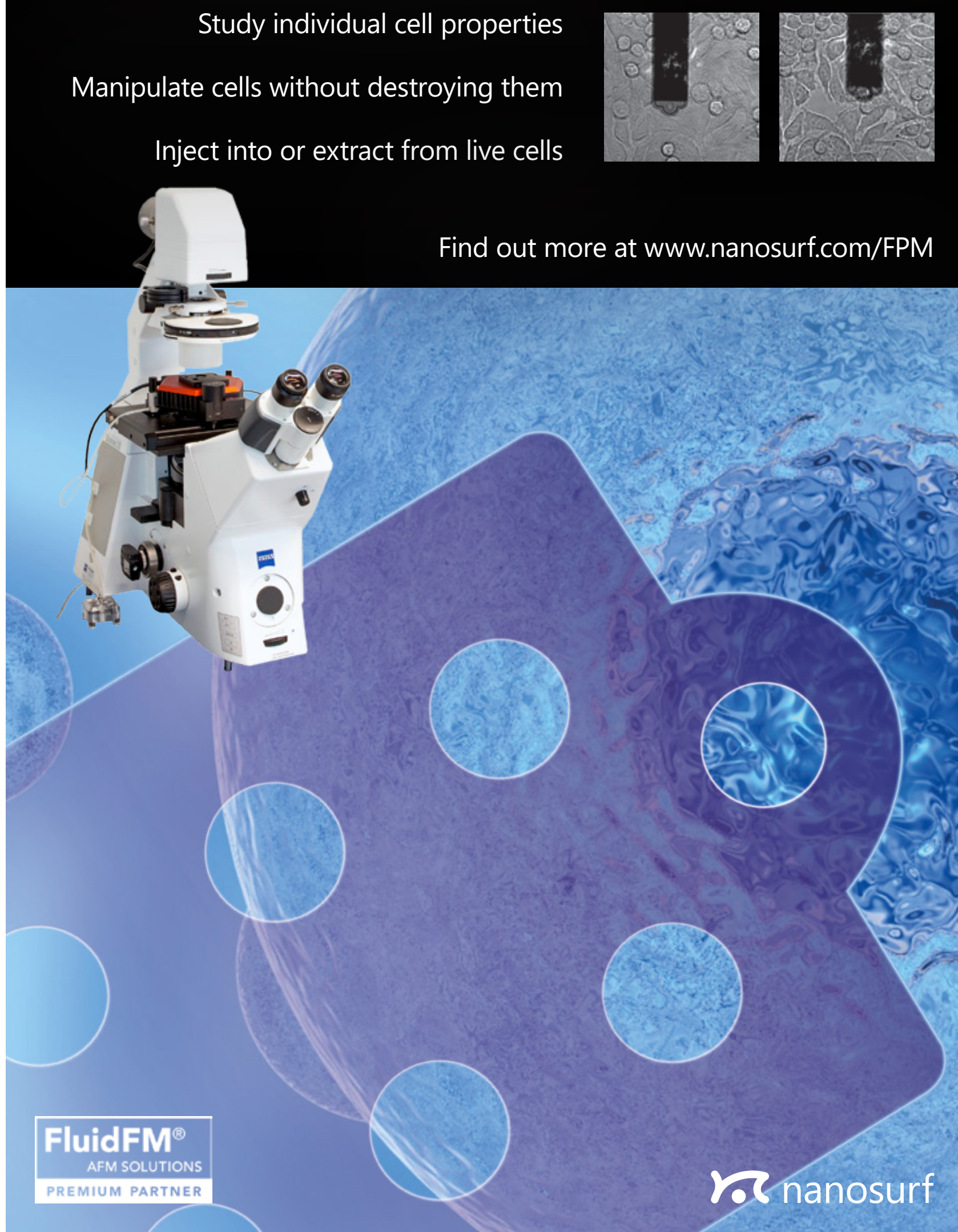